\title{
Mathematical Model and Numerical Analysis of AE Wave Generated by Partial Discharges
}

\author{
D. Wotzka*, T. Boczar And P. Fracz \\ Electric Power Institute, Faculty of Electrical Engineering, Automatic Control and Informatics \\ Opole University of Technology, S. Mikołajczyka 5, 45-271 Opole, Poland
}

\begin{abstract}
Paper presents definition of a mathematical model describing acoustic emission signals generated by partial discharges occurring in oil immersed electric power transformers. Time runs, power spectral density graphs and spectrograms of acoustic emission signals generated in experiments, performed under laboratory conditions, and of equivalent signals, calculated on the basis of the mathematical model, are presented. Furthermore, results of numerical simulations of acoustic pressure distribution inside a tank filled with insulation oil are presented in the paper. In the center of the tank acoustic emission wave sources were placed, each generating signals described by the model developed.
\end{abstract}

PACS: 43.40.Le, 02.60.Lj

\section{Introduction}

One of the common reasons for degradation of oil insulated electric power transformers is connected with existence of partial discharge (PD). Among other methods in the PD diagnosis the acoustic emission method, which is based on measurement and analysis of signals registered by use of acoustic emission (AE) sensors, is frequently applied [1, 2]. Thus propagation phenomena of acoustic waves generated by PDs are currently widely studied $[3-5]$.

The paper contains results of research works aimed to definition of a mathematical model for AE wave source whose acoustic response reflects the real wave generated by $\mathrm{PD}$ that may occur in oil insulated electric power transformer. Authors assumed that the AE signal consists of $N$ impulses of various parameter values, e.g. frequency, time shift, and amplitude. The AE signal modeled was defined at the basis of AE signals generated by PDs in a multi-middle-plate configuration that was mounted in a tank filled with insulation oil containing gas bubbles. The AE signals have been recorded in measurement setups modeling PDs at the Electric Power Institute, Opole University of Technology [3]. Parameters of the model developed by the authors were estimated for compliance with a real AE signal generated by PDs and were evaluated by use of the Nelder-Mead-Simplex method [6].

The further aim of this paper was to present numerical simulations results which regard time-space distribution of acoustic pressure inside a tank filled with electro-insulating oil. The simulations considered time depen-

* corresponding author; e-mail: dzmarzly@gmail.com dent analysis and were performed by use of the COMSOL Multiphysics environment. Five separated AE wave sources were placed in the center of the tank, each generating an acoustic wave as defined by the mathematical model. The signal measured in the far field consisted of interfering waves coming from the particular sources.

\section{Mathematical model of AE wave generated by PDs in insulating oil}

Based on measurements and results analysis of AE signals generated by PD in oil insulation [4] a mathematical model for the AE signal was proposed. In general, the AE signal is generated by a number of single PD pulses, which occur in series (and/or simultaneously), in a small region, close to each other, in a time interval shorter than $1 \mu \mathrm{s}[2,7]$. Duration of the AE signal depends on parameters of the acoustic propagation path and can extend from few milliseconds to one second, while the rise time equals about $10 \mu \mathrm{s}[2]$.

For the research purposes it was assumed that the AE signal $y(t)$ consists of $N$ single PD pulses $y_{i}(t)$, defined by Eq. (1), where the particular PDs differ by: the amplitude $A_{\cos }{ }_{i}$, time offset $\tau_{i}$ and frequency $f_{i}$. The signal (time run) of a single PD is described by the product of a sigmoid function, which depicts the PD rising part, an exponential function, depicting the PD damp down part, and a cosine function for the frequency component

$$
\begin{gathered}
y_{i}(t)=\frac{A}{1+a \mathrm{e}^{-b\left(t-\tau_{i}\right)}} \mathrm{e}^{-\left(\frac{t-\tau_{2}{ }^{i}}{\gamma}\right)} \\
\times A_{\cos \_}{ }^{i \cos \left(2 \pi f_{i}\left(t-\tau_{3}{ }^{i}\right)\right)},
\end{gathered}
$$

where $A$ - amplitude of the sigmoid function [V], $a$ scale coefficient of the sigmoid function [-], $b-$ shape 
coefficient of the sigmoid function [-], $\tau_{1}, \tau_{2}, \tau_{3}-$ localization coefficients $[\mathrm{s}], \gamma$ - shape coefficient of the exponential function [-], $f$ - frequency of the cosines function $[\mathrm{Hz}], A_{\mathrm{cos}}$ - amplitude of the cosine function $[-], t$ - time $[\mathrm{s}]$.

Figure 1 presents time run example of a single PD pulse $f(t)$, modeled by use of Eq. (1).

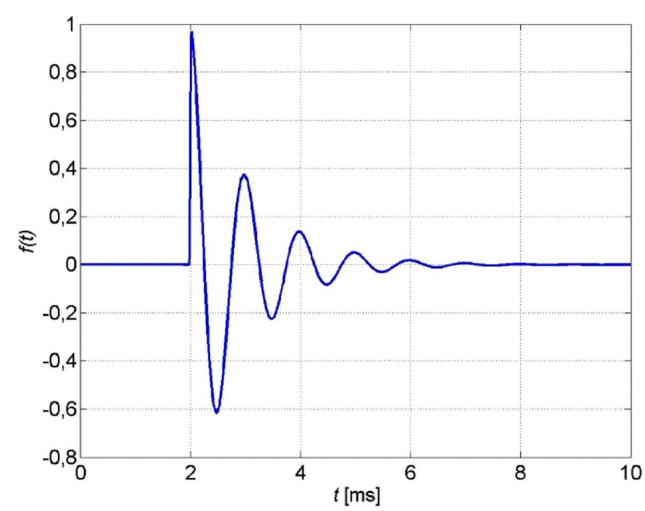

Fig. 1. Time run of a modeled PD pulse.

The rising time of a single $\mathrm{PD}$ pulse is described by the scale coefficient of the sigmoid function. Figure 2 presents chosen time interval depicting the rising part for selected values of the parameter $a$.

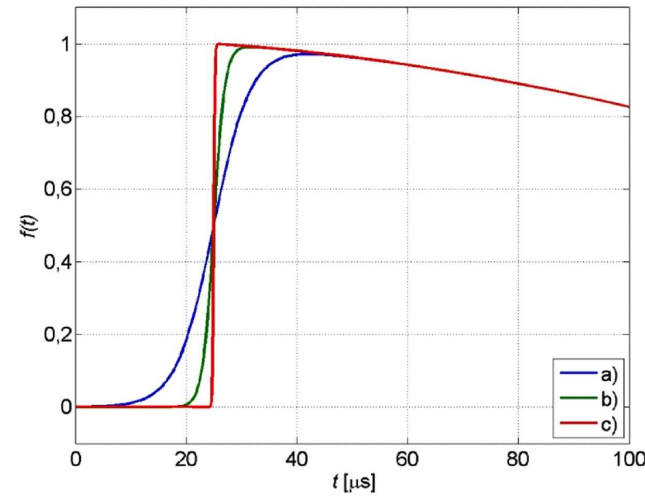

Fig. 2. Chosen time interval of the AE signal generated by single $\mathrm{PD}$, depicting the $\mathrm{PD}$ rising part, for three selected values of $a$ parameter: (a) $a=0.3$, (b) $a=1$, (c) $a=10$.

An acoustic wave falls within the wave phenomena, so the superposition rule was applied and the particular PD pulses were added into one signal, which was written by Eq. (2). It was further assumed that the sigmoid and the exponential functions are the same for all PDs involved in the modeled AE signal

$$
y(t)=\sum_{i=1}^{N} y_{i}(t),
$$

where $N$ - number of PD pulses which are included in the AE signal [-].

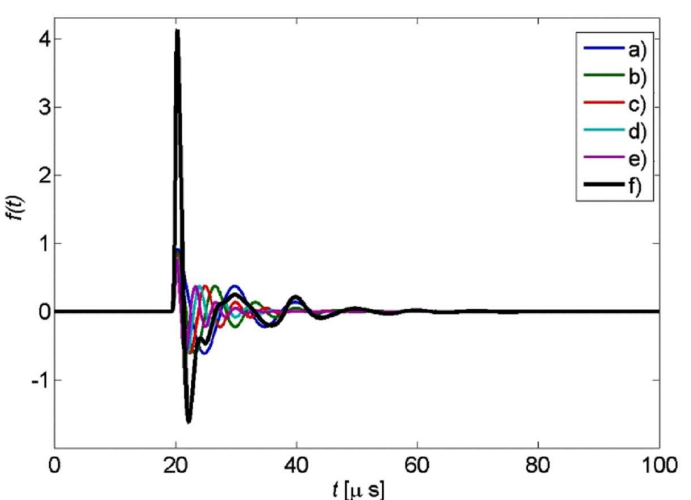

Fig. 3. Time runs of five modeled PD pulses $(a)-(e)$ and the AE signal $(f)$ arising from the superposition rule involved in Eq. (2).

Figure 3 presents time runs of five example PD pulses (Fig. 2a-e), modeled by use of Eq. (1) and time run of AE signal (Fig. 2f) modeled by use of Eq. (2).

\section{Estimation and analysis of model parameters}

For two example signals, which were generated and recorded in a setup equipped with multi needle and plate electrodes immersed in a tank filled with insulation oil, where additionally gas blebs were present, an analysis has been performed in order to evaluate parameters of the model proposed in Eq. (2). Both signal samples (time runs) consisted of 25000 data. It was assumed that the model consists of $N=12 \mathrm{PD}$ pulses. Parameter values have been estimated by use of the Nelder-Mead-Simplex method, supported with MATLAB, complying minimal value of the residual norm $\delta_{\mathrm{R}}(3)$ :

$$
\delta_{\mathrm{R}}=\|\hat{\boldsymbol{y}}-\boldsymbol{y}\|=\sqrt{\sum_{i=1}^{n}\left(\hat{y}_{i}-y_{i}\right)},
$$

where $\delta_{\mathrm{R}}$ - value of the residual norm, $\hat{y}$ - estimate of the regression function, $\boldsymbol{y}$ - empirical data, $i=(1,2, \ldots, n), n=25000$ - number of data in a sample signal.

Such a criterion for the AE signal modeled was chosen to be the most corresponding to the signal measured. As described earlier, the shape of the AE signal is determined by the amplitude, shape, and localization parameters of the sigmoid and exponential functions, while the frequency components and their power are described within the cosine functions frequency and amplitude values. The estimates are listed in Tables I and II.

The comparison analysis of the frequency components and its power $\left(f, A_{\mathrm{cos}}\right)$ regards to power spectral density (PSD) diagrams, which were estimated by use of the Welch method, for two example signals and two signals modeled by use of Eqs. (1) and (2). The mean value of the residual norm was equal to $\delta_{\mathrm{R}}=8.52$. Figure 4 presents power spectral densities for the two analyzed 
TABLE I

Parameter values estimated with the Nelder-MeadSimplex method for the both analyzed signals.

\begin{tabular}{c|c|c|c|c}
\hline \hline$i$ & \multicolumn{2}{|c|}{ Signal no. 1} & \multicolumn{2}{c}{ Signal no. 2} \\
\cline { 2 - 5 }$[-]$ & $A \cos [-]$ & $f[\mathrm{~Hz}]$ & $A \cos [-]$ & $f[\mathrm{~Hz}]$ \\
\hline 1 & 6.70 & 20 & 3.73 & 20 \\
2 & 2.25 & 70 & 1.93 & 70 \\
3 & 3.00 & 120 & 2.98 & 120 \\
4 & 2.54 & 170 & 2.66 & 170 \\
5 & 3.50 & 220 & 4.15 & 220 \\
6 & 4.56 & 270 & 4.29 & 270 \\
7 & -2.0 & 320 & 1.35 & 320 \\
8 & 3.21 & 370 & 1.25 & 370 \\
9 & 2.64 & 420 & 1.07 & 420 \\
10 & 1.11 & 470 & 0.78 & 470 \\
11 & 0.79 & 520 & 0.51 & 520 \\
12 & 0.93 & 570 & 0.49 & 570
\end{tabular}

TABLE II

Parameter values estimated with the Nelder-MeadSimplex method for the both analyzed signals.

\begin{tabular}{c|c|c}
\hline \hline Parameter & Signal no. 1 & Signal no. 2 \\
\hline$A[\mathrm{~V}]$ & $1.5 \times 10^{-5}$ & $3.14 \times 10^{-5}$ \\
$a[-]$ & 0.34 & 0.34 \\
$b[-]$ & 2666 & 2666 \\
$\gamma[-]$ & $2.7 \times 10^{-3}$ & $0.5 \times 10^{-3}$ \\
$\tau_{1}[\mathrm{~s}]$ & 1.7 & 2.8 \\
$\tau_{2}[\mathrm{~s}]$ & 15.0 & 5.1 \\
$\tau_{3}[\mathrm{~s}]$ & 0.0 & 0.0
\end{tabular}

signals recorded under laboratory conditions and their equivalents modeled mathematically. One can recognize that in both signals, measured experimentally, frequency components above $600 \mathrm{kHz}$ occur in a not significant manner, thus the frequency band considered for the signals modeled ends by the value of $f=570 \mathrm{kHz}$, as depicted in Table I.

The comparison analysis of the sigmoid and exponential functions parameters has been performed in the time domain. Figures 5 and 6 present the two considered AE signals time runs and their envelopes, for which the sigmoid and exponential function parameters were determined.

Additionally, a comparison analysis has been performed in the time-frequency domain by use of the short time Fourier transform (STFT). Figures 7 and 8 present the time-frequency structures estimated for the signals: recorded under laboratory conditions (on the left) and calculated on the basis of the model developed (on the right), for the both signals analyzed.

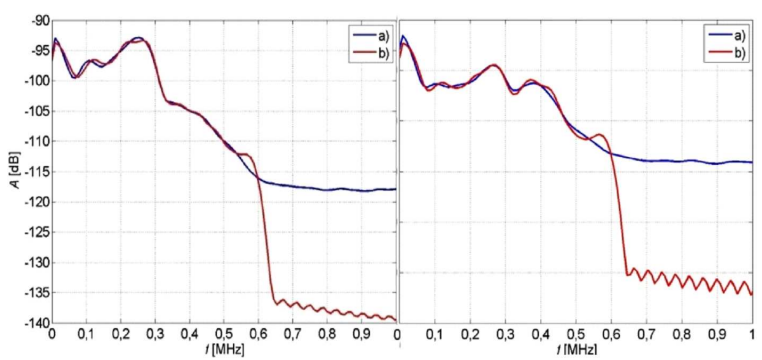

Fig. 4. PSD diagram for AE signals recorded in experiments $(a)$ and mathematically $(b)$. Left: signal no. 1, right: signal no. 2 .

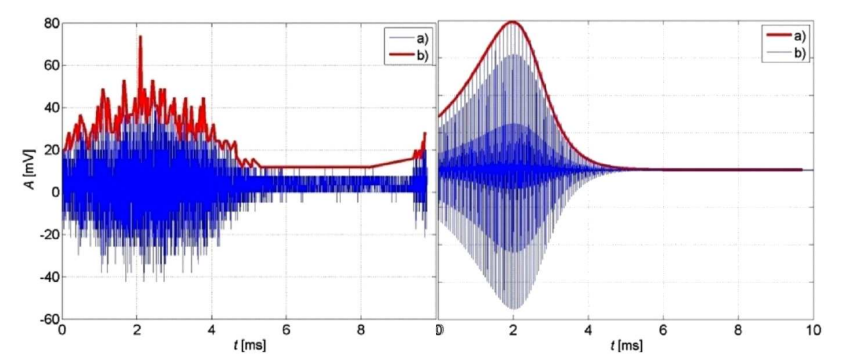

Fig. 5. Time runs and envelopes of AE signals recorded in experiment (left) and calculated mathematically (right). Signal no. 1.

On the basis of comparison analysis performed in the time, frequency and time-frequency domains for two exemplary AE signals measured under laboratory conditions, it was stated that it is possible to determine parameters of the model proposed in Eqs. (1) and (2) for good agreement between the signals recorded and calculated.

\section{Numerical modeling of AE wave}

In the scope of research works performed the developed mathematical model of $\mathrm{AE}$ wave generated by $\mathrm{PD}$ was applied as acoustic wave source in a numerical simulation. The simulation considers acoustic pressure distribution inside a tank filled with electro-insulation oil (Fig. 9) in center of which the AE wave source is placed. The mathematical model, given by Eq. (2), consists of

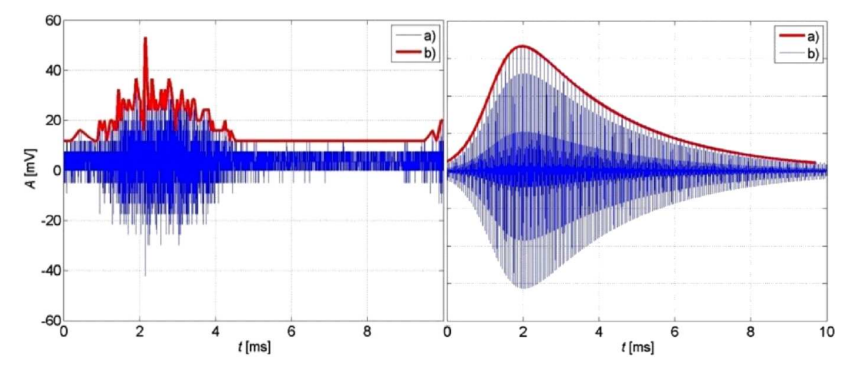

Fig. 6. As in Fig. 5, but for signal no. 2. 


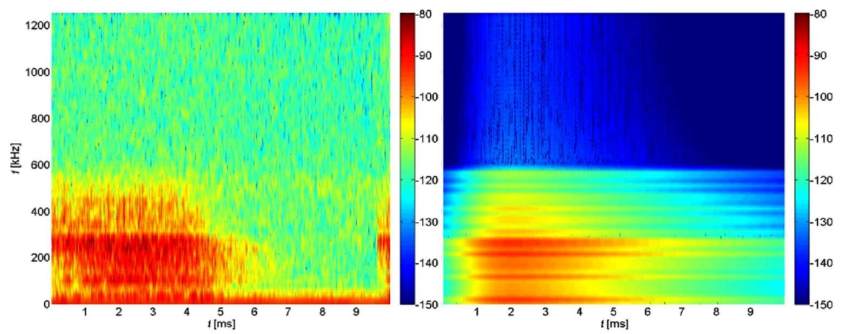

Fig. 7. Spectrograms of AE signals recorded in experiment (left) and calculated mathematically (right). Signal no. 1 .

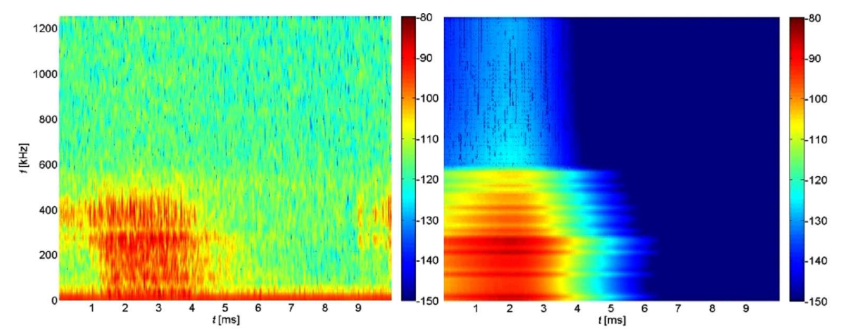

Fig. 8. As in Fig. 7, but for signal no. 2.

$N=5$ single PD sources, each emitting AE wave, as described by Eq. (1).

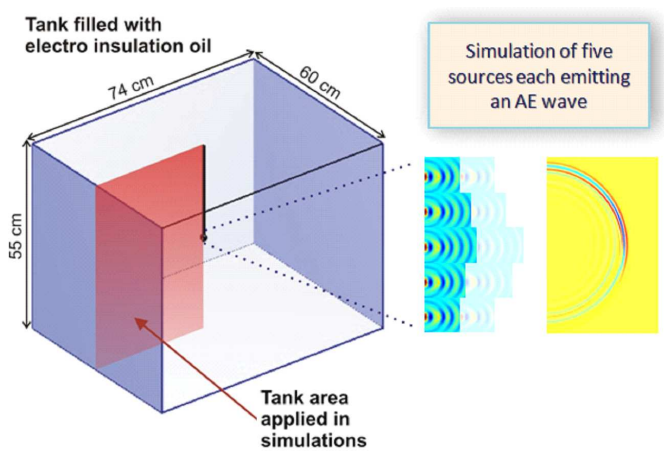

Fig. 9. Geometry of the object considered in numerical simulations, a tank filled with oil, depicting five AE wave sources placed in the central part of the tank.

Due to limitations in the author's equipment (Intel Core2Quad processor and 4 GB of RAM) the model has been reduced to two dimensions and further an existing axial symmetry has been applied. The two-dimensional model of the tank, applied in simulations, is presented in Fig. 10.

The numerical model does not consider any physical phenomena except the acoustical wave propagation. Thus neither damping nor attenuation coefficients have been involved in calculations. Further it was assumed that the acoustic wave propagates in an ideal fluid, with constant temperature, density and speed of sound values. The wave equation calculated numerically is presented by Eq. (4). The dependent variable regards to

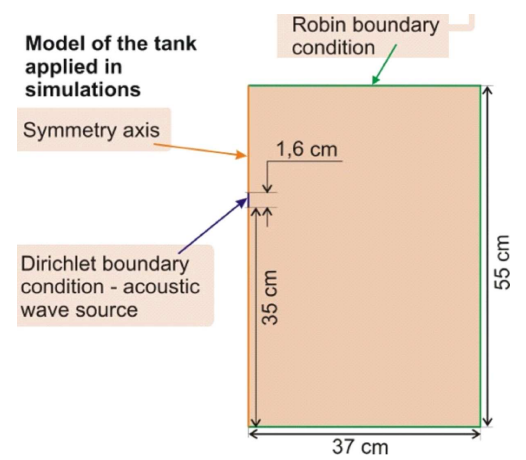

Fig. 10. Geometry of the two-dimensional tank applied in simulations and the boundary conditions assumed.

acoustic pressure $p$. The Laplace operator is applied on two independent variables - space dimensions; the third independent variable is the time

$$
\nabla \cdot\left(\frac{-1}{\rho_{0}} \nabla p\right)+\frac{1}{c^{2} \rho_{0}} \frac{\partial^{2} p}{\partial t^{2}}=0,
$$

where $\rho_{0}$ - density $\left[\mathrm{kg} / \mathrm{m}^{3}\right], p-$ acoustic pressure [Pa], $t$ - time $[\mathrm{s}], c$ - sound speed $[\mathrm{m} / \mathrm{s}]$.

The boundary conditions assumed in simulations were the following (see also Fig. 10):

1) Robin boundary on the tank surfaces

$$
n \cdot\left(\frac{1}{\rho_{0}} \nabla p\right)+\frac{1}{Z} \frac{\partial p}{\partial t}=0, \quad Z=123.7\left[\frac{\mathrm{kg}}{\mathrm{m}^{3}}\right] .
$$

2) Dirichlet boundary for each $\mathrm{AE}$ wave source $p=$ $y_{i}(t)$, as given by Eq. (1).

3) Symmetry boundary on the axial symmetry.

The tank was assumed to be filled with electro-insulation oil of density equal to $\rho=890 \mathrm{~kg} / \mathrm{m}^{3}$ and speed of sound equal to $c_{\mathrm{s}}=1390 \mathrm{~m} / \mathrm{s}$. For all five $\mathrm{AE}$ wave sources the same value of the sigmoid and the exponential function parameters have been assumed. The acoustic sources differed by value of the frequency component. Parameter values used in simulation are presented in Table III.

TABLE III

Parameter values of the five AE wave sources modeled and applied in numerical simulations.

\begin{tabular}{c|c|c|c}
\hline \hline Parameter & Value & Parameter & Value \\
\hline$A\left[\mathrm{~m}^{3} / \mathrm{s}\right]$ & $100 \times 10^{3}$ & $f_{1}$ & 100 \\
$a[-]$ & 1 & $f_{2}$ & 150 \\
$b[-]$ & $1 \times 10^{8}$ & $f_{3}$ & 200 \\
$\gamma[-]$ & $1 / f$ & $f_{4}$ & 250 \\
$\tau[\mathrm{s}]$ & 0 & $f_{5}$ & 300
\end{tabular}

The numerical simulations have been performed by use of the acoustic pressure module supported within 
COMSOL Multiphysics, which applies the finite element method. The object was discretized with a mesh of a single element not bigger than $2.78 \mathrm{~mm}$, which resulted with 22919 DoF. It should be noted that the simulation described does not concern the surface waves emerging as mechanical vibrations at the tank surface.
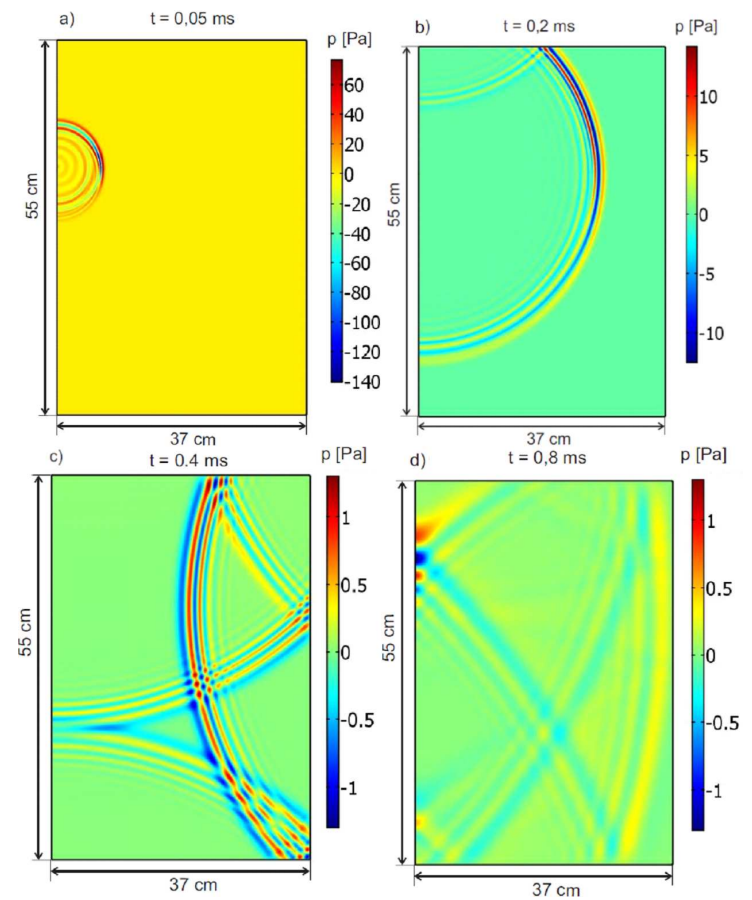

Fig. 11. Acoustic pressure distributions inside the object under study calculated at following simulation times: (a) $t=0.05 \mathrm{~ms}$, (b) $t=0.2 \mathrm{~ms}$, (c) $t=0.4 \mathrm{~ms}$, (d) $t=0.8 \mathrm{~ms}$.

Figure 11 presents acoustic pressure distribution inside the two-dimensional model of the tank at selected simulation times. One can recognize that the AE waves generated by the particular sources interfere in a series of AE waves (Fig. 11a), which propagate as spherical waves (Fig. 11c). The wave reflects from the tank surface (Fig. 11c, d), which results from the Robin boundary. Due to distribution of energy inside the tank and due to the impedance boundary on the tank surface the wave amplitude $(p[\mathrm{~Pa}])$ decreases with time (Fig. 11d, e).

\section{Summary}

Definition of a mathematical model for AE signals generated by $\mathrm{PD}$ in oil insulated power transformers has been presented in the paper. Parameter values have been estimated numerically with the Nelder-Mead-Simplex method based on signals measured under laboratory conditions. Results of a comparison analysis regarding time, frequency and time-frequency domains for signals measured and modeled mathematically are discussed. On the basis of the time runs, power spectral densities and spectrograms presented, it was stated that it is possible to evaluate parameter values of the model defined, so that it complies with the AE signals generated by PD and measured in laboratory experiments. The mathematical model has been further applied in numerical simulations as AE source. Acoustic pressure distribution inside a two-dimensional tank filled with insulation oil was discussed in the paper. Further research will aim to numerical simulations of acoustic pressure distribution inside a three-dimensional object and a practical verification, where a wide band acoustic wave generator (a piezoelectric transducer) will be applied for generation of an $\mathrm{AE}$ signal as described by the mathematic model in Eqs. (1) and (2).

\section{References}

[1] F. Witos, A. Olszewska, Acta Phys. Pol. A 118, 1267 (2010).

[2] J. Skubis, Acoustical Emission in Investigations of Isolators Systems, IPPT-PAN, Warsaw 1993 (in Polish).

[3] D. Wotzka, Ph.D. Thesis, Opole University of Technology, 2011 (in Polish).

[4] D. Wotzka, T. Boczar, D. Zmarzły, Acta Phys. Pol. A 116, 428 (2009).

[5] D. Wotzka, T. Boczar, D. Zmarzły, Acta Phys. Pol. A 118, 1272 (2010).

[6] J.C. Lagarias, J.A. Reeds, M.H. Wright, P.E. Wright, SIAM J. Optim. 9, 112 (1998).

[7] A. Opilski, I. Malecki, Characteristics and Classification of Acoustical Emission Signals, Ed. J. Ranachowski, IPPT-PAN, Warsaw 1994 (in Polish). 